\title{
Síndrome de amok en un cuadruple crimen, acting out e impulsividad patológica
}

\section{Amok syndrome in a quadruple crime, acting out and pathological impulsivity}

\author{
DOI: $10.46932 / \mathrm{sfjdv} 2 \mathrm{n} 4-039$
}

Received in: May 1st, 2021

Accepted in: Jun 30th, 2021

\author{
Dr. Bernat-Noël Tiffon Nonis \\ Universitat Abad Oliba-CEU. Calle de Bellesguard, no30. 08022 Barcelona (Spain). \\ E-mail: btiffonn@uao.es
}

\section{RESUME}

Se describe y repasa el concepto de Síndrome de Amok y se describe un caso de asesinato de 4 víctimas perpetrado por un sujeto que, dejándose embargar por su arrebato e ira, comete los hechos delictivos criminológicos.

Palabras clave: Síndrome de Amok, Impulsividad Patológica, Asesinatos, Acting Out, Trastorno Explosivo Intermitente, cuádruple asesinato.

\section{ABSTRACT}

The concept of Amok Syndrome is described and reviewed, and a case of murder of 4 victims perpetrated by a subject who, being seized by his rage and anger, commits the criminological criminal acts is described.

Keywords: Amok Syndrome, Pathological Impulsivity, Murders, Acting Out, Intermittent Explosive Disorder, quadruple murder.

\section{INTRODUCCIÓN}

Según el DSM-IV (1995), el Amok se caracteriza por un episodio agudo de comportamiento violento y descontrolado que la persona no recuerda posteriormente. A diferencia del Trastorno Explosivo Intermitente, el Amok se presenta como un episodio único más que como un patrón de comportamiento agresivo y a menudo está asociado a rasgos disociativos prominentes. Los episodios de comportamiento violento son más frecuentes en sexo masculino que en femenino.

Asimismo, y aunque se observa tradicionalmente en los países del sudeste asiático, también se han presentado casos de Amok en Canadá y Estados Unidos.

El propio Manual DSM-IV (1995), considera el Amok como de un Trastorno Explosivo Intermitente, pero de prevalencia rara definiéndolo como un episodio disociativo caracterizado por un período de depresión seguido de una manifestación de comportamiento violento, agresivo u homicida, dirigido a personas y objetos. El episodio se desencadena por la percepción de una falta de respeto o de un insulto y parece ser prevalente entre los varones. El episodio se acompaña frecuentemente de ideas 
paranoides, automatismo, amnesia, agotamiento y retorno al estado premórbido tras el episodio. En algunas ocasiones, el Amok puede aparecer durante la presentación de un episodio psicótico breve o constituir el comienzo o una exacerbación de un proceso psicótico crónico. Los informes originales que utilizaban este término eran de Malasia. Para Esteban (2010), su origen es tan antiguo que ya apareció citada en un libro del portugués Duarte Barbosa escrito en el siglo XVI, donde este comerciante que viajó con la expedición de Magallanes relató el comportamiento de algunos habitantes de la isla indonesia de Java: “que salían a la calle y mataban a tantas personas como se encontraban”. Según los especialistas, "el individuo que lo padece tras sufrir una fuerte vergüenza social; empieza a correr sin cesar destrozando todo lo que encuentra a su paso, incluso matando los animales y a las personas que se tropiezan en su camino. Normalmente el resto de la población lo acaba matando por ser un ser peligroso, pero los que llegan a sobrevivir, muestran una amnesia total sobre lo ocurrido. Podría sugerirse que se trata de un ataque de pánico, esquizofrenia o una paranoia transitoria pero la verdad es que los especialistas occidentales no se ponen de acuerdo en la naturaleza exacta del amok".

Un patrón de comportamiento similar se encuentra en Laos, Filipinas, Polinesia (cafard o cathard), Papúa-Nueva Guinea y Puerto Rico (mal de pelea), y entre la población navaja (iich'aa).

En cuanto al curso de dicha disfunción, existen pocos datos sobre la edad de comienzo del trastorno explosivo intermitente, pero puede aparecer desde la adolescencia tardía hasta la tercera década de la vida. Y, la forma de presentación es brusca y sin período prodrómico.

Para proceder al diagnóstico de dicha disfuncionalidad, el diagnóstico de Trastorno Explosivo Intermitente debe considerarse únicamente después de que hayan sido descartados todos los trastornos que van asociados a comportamientos impulsivos o agresivos.

Cabe señalar, tal como bien dice el propio Manual DSM-IV (1995), son útiles la práctica de una detallada historia clínica y una completa exploración neurológica para establecer el pronóstico. Las anomalías inespecíficas de la exploración neurológica y los cambios EEG inespecíficos son compatibles con el diagnóstico de trastorno explosivo intermitente siempre que no sean resultado de una enfermedad médica diagnosticable.

El Trastorno Explosivo Intermitente debe distinguirse del comportamiento agresivo o errático que pueden manifestar sujetos con trastorno negativismo desafiante, el trastorno disocial, el trastorno antisocial de la personalidad, el trastorno límite de la personalidad, un episodio maníaco y la esquizofrenia. Es por ello que, si el comportamiento agresivo se explica mejor por la presencia de otro trastorno mental, no se debe realizar el diagnóstico de trastorno explosivo intermitente.

En este sentido, en el Síndrome de Amok el comportamiento agresivo puede aparecer en ausencia de un trastorno mental. El comportamiento finalista se distingue del trastorno explosivo intermitente por 
la existencia de incentivos y ganancias con el acto agresivo. En el contexto forense las personas pueden simular un trastorno explosivo intermitente para evitar la responsabilidad derivada de su comportamiento (DSM-IV, 1995).

Para el DSM-IV (1995) los criterios para el diagnóstico de F63.8 Trastorno explosivo intermitente [312.34] son los siguientes:

A. Varios episodios aislados de dificultad para controlar los impulsos agresivos, que dan lugar a violencia o a destrucción de la propiedad.

B. El grado de agresividad durante los episodios es desproporcionado con respecto a la intensidad de cualquier estresante psicosocial precipitante.

C. Los episodios agresivos no se explican mejor por la presencia de otro trastorno mental (p. ej., trastorno antisocial de la personalidad, trastorno límite de la personalidad, trastorno psicótico, episodio maníaco, trastorno disocial o trastorno por déficit de atención con hiperactividad) y no son debidos a los efectos fisiológicos directos de una sustancia (p. ej., drogas, medicamentos) o a una enfermedad médica (p. ej., traumatismo craneal, enfermedad de Alzheimer).

En el presente caso, se trata de un varón de 58 años de edad, natural de un municipio de la provincia de Girona y de estado civil soltero. Ocupa el $1^{\text {er. }}$ puesto de una fratría de 3 hermanos. Su nivel académico es básico, llegando a alcanzar el Certificado de Estudios Primarios. Su trayectoria profesional la ha venido ejerciendo a lo largo de unos 28-30 años como albañil. Como antecedentes psicopatológicos clínicos, el informado refirió que cuando disponía la edad de 8-9 años, sufrió un traumatismo en la cabeza por accidente de bicicleta (añade que fue intervenido pero que no dispone documentación clínica que se recoja dicha actuación profesional sanitaria dado el tiempo transcurrido).

El informado fue visitado como interno preventivo en un centro penitenciario cercano de la provincia anteriormente mencionada a la espera de ser enjuiciado por un delito de 4 asesinatos (cuádruple crimen). El Íter Criminis del informado fue el siguiente:

- 2 asesinatos en un bar de un municipio de la provincia de Girona. Las víctimas eran padre e hijo y fueron asesinados a tiros de escopeta.

- 2 asesinatos perpetrado al cabo de unos 15 minutos (aproximadamente) en una caja de ahorros de un municipio cercano al anterior de la misma provincia y por cuya distancia entre uno y otro era de unos $11 \mathrm{Km}$ (aproximadamente). Las víctimas eran trabajadores de dicha sucursal bancaria. 
Figuras 1 y 2: Reconstrucción de los hechos.
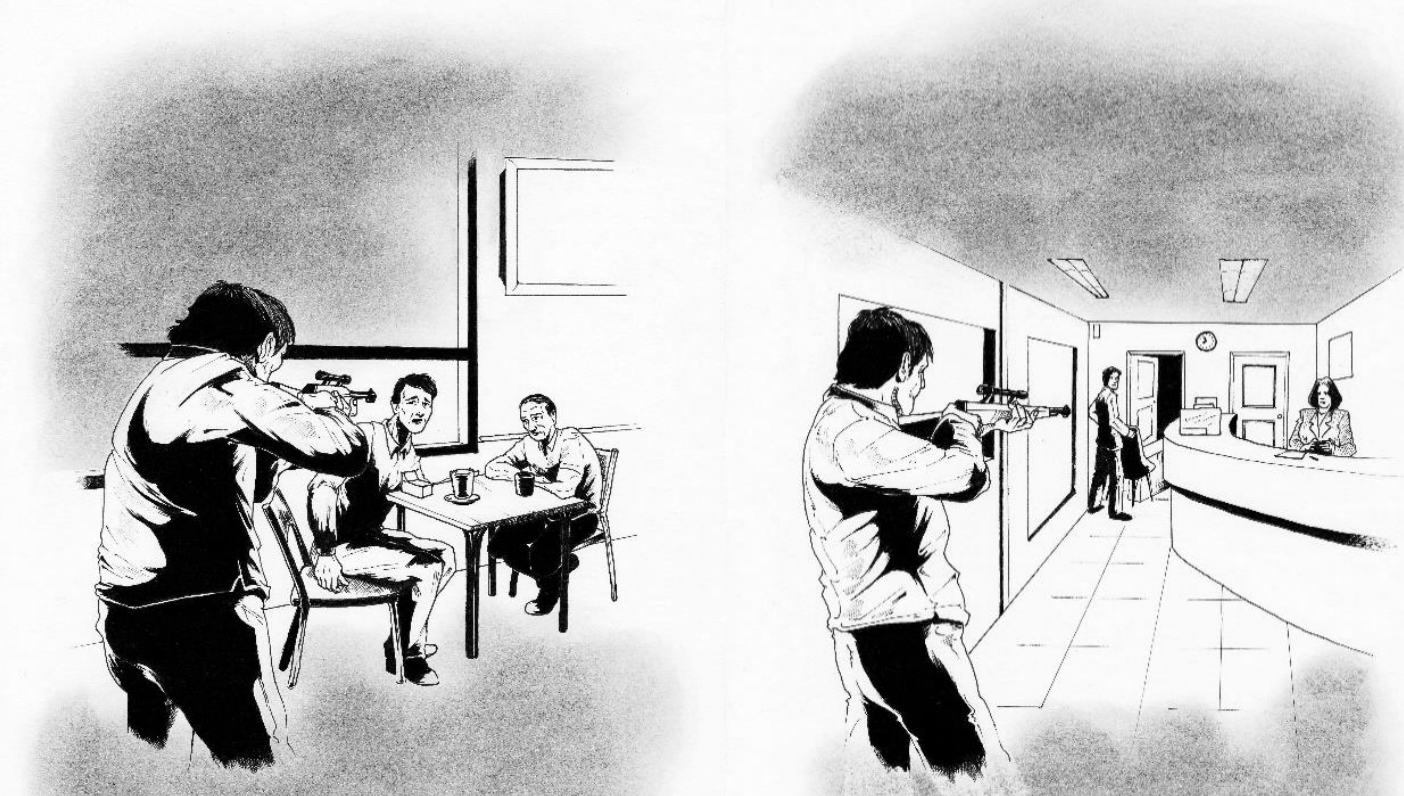

Ilustraciones de Juan Antonio Valverde. En Tiffon, N.-N. (2015). “Los Crímenes de Perejil”. Bosch Editor. Barcelona.

\section{RESULTADOS:}

Para el desarrollo del estudio de la personalidad del informado se procedió a la siguiente metodología:

1. Entrevista clínico-pericial dirigida (anamnesis) con el informado con una inversión total de horas aproximadas de 4h. en un Centro Penitenciario de la provincia de Barcelona.

2. Entrevista con la hermana del informado.

3. Administración actualizada de pruebas psicométricas con objeto a evaluar su estado psíquico y psicopatológico (MMPI-2; MCMI-3, 16PF, TCI-R, Cuestionario de Salamanca, STAI, BISS-11 de impulsividad patológica, EMCA, MEC de Folstein y Cuestionario Pfeiffer).

4. Análisis de la documentación clínica y jurídica aportada.

A raíz de la administración de los cuestionarios psicopatológicos y de las distintas entrevistas clínicas dirigidas celebradas, los resultados obtenidos vienen a sugerir que el informado (perpetrador de las agresiones) presentaba compatibilidad con un Trastorno Explosivo Intermitente con modalidad subclínica de impulsividad patológica de Síndrome de Amok en los precisos momentos e instantes de la 
perpetración de los hechos acaecidos y que fueron motivo de debate jurídico-legal (tanto en el bar como en la caja de ahorros).

\section{DISCUSIÓN/CONCLUSIONES:}

En el contexto de exploración y análisis psicopatológico y criminológico del Íter Criminis perpetrado por el agresor y por cuyo resultado de muerte fue de 4 víctimas adultas (3 hombres y una mujer), el informado presentaba compatibilidad de disfunción psíquica de impulsividad patológica originariamente mal canalizada por la rabia y cólera que el mismo presentaba al no tolerar la frustración de no ser debidamente remunerado profesionalmente (Tiffon, 2015). Asimismo, y al cabo de unos 15 minutos de perpetrarse el primer acto criminalmente impulsivo en acting out, el perpetrador tampoco tolera su frustración al reclamar un importe endeudado por la caja de ahorros y perpetra su segundo acto criminal profiriendo la muerte de 2 personas más en el acto con arma de fuego (escopeta).

La conducta delictivo-criminológica fue el producto de un conjunto de factores psicopatológicos de base que actuaron como condicionantes previos en la materialización conductual anteriormente descrita:

- Impulsividad patológica (entendido como Trastorno del Control de los Impulsivos, submodalidad de Trastorno Explosivo Intermitente).

- Trastorno Mixto de la Personalidad.

- Deterioro neurocognitivo por factor de la edad y escaso nivel académico-formativo. 


\section{REFERENCIAS}

- American Psychiatric Association (1995). "Diagnostic and Statistical Manual of Mental Disorders: DSM-IV. Washington. Versión española de la cuarta edición de la obra original en lengua inglesa. 1995. MASSON, S.A. Barcelona (España).

- $\quad$ Esteban, M. (2010). “Geografía del desarrollo humano”. Editorial Aresta. Barcelona.

- $\quad$ Tiffon, B.N. (2015). “Los Crímenes De Perejil”. J.M. Bosch Editor. Barcelona.

- $\quad$ Tiffon, B.-N. (2017). “Atlas de Psicología Forense (Penal)”. J.M. Bosch Editor. Barcelona.

- $\quad$ Tiffon, B.-N. y Cols. (2019). “Atlas Práctico-Criminológico De Psicometría Forense (Volumen I): Asesinatos”. J.M. Bosch Editor. Barcelona.

- $\quad$ Tiffon, B.-N. y Cols. (2019). “Atlas Práctico-Criminológico De Psicometría Forense (Volumen II): Tentativas De Asesinatos”. J.M. Bosch Editor. Barcelona.

- $\quad$ Tiffon, B.-N. (2021). "Amok syndrome in a quadruple crime, acting out and pathological drive.". Vol. 2 No. 2 (2021): South Florida Journal of Development, Miami, v. 2, n. 2, apr./jun. 2021 ISSN: 26755459. Págs. 3549-3554. DOI: https://doi.org/10.46932/sfjdv2n2-189

- $\quad$ Tiffon, B.-N. (2021). "Criminal personality in a trained elite military and assassination". Vol. 2 No. 2 (2021): South Florida Journal of Development, Miami, v. 2, n. 2, apr./jun. 2021 ISSN: 2675-5459. Págs. 3555-3559. DOI: https://doi.org/10.46932/sfjdv2n2-190

- $\quad$ Tiffon, B.-N- (2021). "Murder induced deliriously by a "folie à deux". Vol. 2 No. 2 (2021): South Florida Journal of Development, Miami, v. 2, n. 2, apr./jun. 2021 ISSN: 2675-5459. Págs. 3560-3564. DOI: https://doi.org/10.46932/sfjdv2n2-191

- $\quad$ Tiffon, B.-N. (2021). "Double filicide for extended suicide (frustrated) of a subject with major psychotic depression and dependent personality disorder". South Florida Journal of Development, Miami, v. 2, n. 3, Special Edition, jul. 2021. Págs. 4552-4562. DOI: https://doi.org/10.46932/sfjdv2n3-057

- $\quad$ Tiffon, B.-N. (2021). "Delusional psychotic disorder, substance use and murder with alevosia and cruelty". South Florida Journal of Development, Miami, v.2, n.3, p.4544-4551 Special Edition, jul. 2021. Págs. 4544-4550. DOI: https://doi.org/10.46932/sfjdv2n3-056

- $\quad$ Tiffon, B.-N. (2021). "Criminal and Forensic Psychology of a Case of Filicide by Decapitation of a Minor". Journal of Forensic Medicine. Case Series. Volume 6:3. ISSN: 2472-1026 
- $\quad$ Tiffon, B.-N. \& González-Fernández (2021). "Amok Syndrome. Qualified Perspectives on an Aggressive Reaction of Pathological Impulsiveness in The Perpetration of a Double Crime with a Firearm". ASEAN Journal of Psychiatry, Vol. 22(S2), June 2021: 1-5. ISSN: 2231-7791

- $\quad$ Tiffon, B.-N. \& González-Fernandez, J. (2021). Alcohol Consumption and Intensification of Paranoid Traits in Attempted Homicide. ASEAN Journal of Psychiatry, Vol. 22(4), June 2021: 1-5. ISSN: 2231-7791

- $\quad$ Tiffon, B.-N. \& González-Fernández. J. (2021). "Cocaine Cravings, Borderline Personality Disorder \& Attempted Homicide". ASEAN Journal of Psychiatry, Vol. 22(5), July 2021; 1-4. ISNN: 22317791 - Págs. 1-4 